\title{
A unified view of determinantal expansions for Jack Polynomials
}

\author{
Leigh Roberts $^{1}$ \\ School of Economics and Finance \\ Victoria University, P. O. Box 600 \\ Wellington, New Zealand \\ leigh.roberts@vuw.ac.nz
}

Submitted: September 28, 2000; Accepted: December 29, 2000

AMS Subject Classification: $05 E 05$

\begin{abstract}
Recently Lapointe et. al. [3] have expressed Jack Polynomials as determinants in monomial symmetric functions $m_{\lambda}$. We express these polynomials as determinants in elementary symmetric functions $e_{\lambda}$, showing a fundamental symmetry between these two expansions. Moreover, both expansions are obtained indifferently by applying the Calogero-Sutherland operator in physics or quasi Laplace Beltrami operators arising from differential geometry and statistics. Examples are given, and comments on the sparseness of the determinants so obtained conclude the paper.
\end{abstract}

\section{Introduction}

\subsection{Notation}

The partition $\lambda$ has weight $w(\lambda)$. The conjugate partition to $\lambda$ is denoted by $\lambda^{\prime}$, while the transpose of a matrix $A$ is denoted by $A^{T}$. Variates are denoted by $x_{1}, x_{2}, \ldots, x_{n}$, with $D_{i}=\partial / \partial x_{i} ;$ and $w=w(\lambda)=w(\kappa)=\ldots$ throughout.

The dominance (partial) ordering is denoted by $\leq$ : thus $\kappa=\left(k_{1}, k_{2}, \ldots\right) \leq \lambda=$ $\left(l_{1}, l_{2}, \ldots\right) \Leftrightarrow k_{1}+k_{2}+\ldots+k_{i} \leq l_{1}+l_{2}+\ldots+l_{i}$ for all $i$. The conventional total

\footnotetext{
${ }^{1}$ Thanks are due to Peter Forrester for commenting on an early draft, and an anonymous referee who simplified the structure of the paper and suggested improved proofs of Lemma 7 and Theorem 8. Any errors remain the responsibility of the author.
} 
ordering of partitions, viz. the reverse lexicographic ordering (RLO), is denoted by $\stackrel{R}{\leq}$ : hence $(4) \stackrel{R}{\geq}(3,1)$.

The $m_{\lambda}$ functions are stacked into a column vector $M$ in RLO, so that the ordering of the indices of the vector elements from the top is $(w),(w-1,1),(w-2,2),(w-2,1,1), \ldots$. There is an analogous stacking of the $e_{\lambda}$ functions into a column vector $E$.

\subsection{Overview}

In [3] the Calogero-Sutherland operator is given by Lapointe et. al. as:

$$
\mathcal{H}^{(\alpha)}=\mathcal{H}=\frac{\alpha}{2} \sum_{i=1}^{n}\left(x_{i} D_{i}\right)^{2}+\frac{1}{2} \sum_{\substack{i, j=1 \\ i<j}}^{n}\left(\frac{x_{i}+x_{j}}{x_{i}-x_{j}}\right)\left(x_{i} D_{i}-x_{j} D_{j}\right) .
$$

Following Stanley [6, p. 84], we define the operator:

$$
\mathcal{L}^{(\alpha)}=\mathcal{L}=\frac{\alpha}{2} \sum_{i=1}^{n} x_{i}^{2} D_{i}^{2}+\sum_{\substack{i, j=1 \\ i \neq j}}^{n} \frac{x_{i}^{2}}{x_{i}-x_{j}} D_{i}
$$

which we shall call the quasi Laplace Beltrami operator. The operators will be denoted by $\mathcal{H}$ and $\mathcal{L}$ except in $\S 3$ where the more precise notation $\mathcal{H}^{(\alpha)}$ and $\mathcal{L}^{(\alpha)}$ is called for.

When $\alpha=2$, the operator $\mathcal{L}$ is the Laplace Beltrami operator of differential geometry for $G L(n, R)$ acting on cosets of the orthogonal group $O(n)$ (see i.a. [1, p. 386], [2]). For $\alpha=1$ the Jack Polynomials correspond to the Schur functions, while in the case $\alpha=1 / 2$ they provide zonal polynomials for the skew-field of the quaternions ([4, pp. 440, 446]).

A comparison of (1) and (2) soon reveals the first half of the relation

$$
\mathcal{H}-\mathcal{L}=\frac{\alpha-n+1}{2} \sum_{i} x_{i} D_{i}=\frac{w}{2}(\alpha-n+1) .
$$

For symmetric homogeneous polynomials, the operator $\sum_{i} x_{i} D_{i}$ amounts to multiplication by $w$, as is most easily seen by using as basis the power symmetric functions $p_{\lambda}$ (for the definition of which see e.g. [6, p. 77], [4, p. 23]). For the purposes of this paper, then, $\mathcal{H}$ and $\mathcal{L}$ differ by a constant, given in the second half of $(3)$.

This paper encompasses the basic relations (4), (5), (6) and (7).

$$
\mathcal{H} J_{\lambda}=d_{\lambda} J_{\lambda} \quad \mathcal{L} J_{\lambda}=c_{\lambda} J_{\lambda} .
$$

THE ELECTRONiC JOURNAL OF COMBINATORICS 8 (2001), \#R3 
The equations in (4) are to be found in [3] and [6, p. 77] respectively, with eigenvalues given explicitly in (8) and (9).

The relation $\mathcal{L} M=\Omega^{l m} M$ breaks down into:

$$
\mathcal{L} m_{\lambda}=c_{\lambda} m_{\lambda}+\sum_{\lambda>\kappa}\left(\Omega_{u}^{m}\right)_{\lambda \kappa} m_{\kappa}
$$

Lapointe et. al. [3] work out $\mathcal{H} m_{\lambda}$, and find an equation of the form of (5) except that $c_{\lambda}$ is replaced by $d_{\lambda}$. From [3] we know that $\Omega_{u}^{m}$ is upper triangular with zero diagonal, and in addition does not depend on $\alpha$.

In similar vein $\mathcal{L} E=\Omega^{l e} E$ decomposes into:

$$
\mathcal{L} e_{\lambda}=c_{\lambda^{\prime}} e_{\lambda}+\sum_{\kappa>\lambda}\left(\Omega_{l}^{e}\right)_{\lambda \kappa} e_{\kappa}
$$

Again, for the application of $\mathcal{H}$ to $e_{\lambda}$, the only alteration to the right side of (6) would be a change of $c_{\lambda^{\prime}}$ to $d_{\lambda^{\prime}}$. One of the main aims of this paper is to deduce the form of $\Omega_{l}^{e}$ from that of $\Omega_{u}^{m}$, which is given in the next equation.

$$
\Omega_{l}^{e T}=-\alpha \Omega_{u}^{m},
$$

thereby justifying the suffix $l$, since $\Omega_{l}^{e}$ is lower triangular.

From (4), $J_{\lambda}$ is an eigenfunction of both operators, and the cited papers providing the form of the operators in (1) and (2) also provide the corresponding eigenvalues:

$$
\begin{gathered}
c_{\lambda}=\left(n-\frac{\alpha}{2}\right) w-\sum j l_{j}+\frac{\alpha}{2} \sum l_{j}^{2} \\
d_{\lambda}=c_{\lambda}-\frac{w}{2}(n-\alpha-1) .
\end{gathered}
$$

The difference between the eigenvalues naturally mirrors that between the operators in (3), and depends on $\lambda$ only through its weight.

The determinantal expression for $J_{\rho}$ in terms of the $m_{\lambda}$ functions is given in [3]. Related work was done in [5] with both basis functions for the special case of zonal polynomials $(\alpha=2)$, although results were not expressed as determinants.

In $\S 2$ the expansions of $J_{\rho}$ as determinants in $e_{\lambda}$ and $m_{\lambda}$ functions are given. The basic symmetry between these expansions is exhibited in $\S 3$, in which (7) is proved. The remaining technical work in the paper is the proof that the coefficient of $e_{\lambda}$ in (6) is indeed $c_{\lambda^{\prime}}$, which is undertaken in Theorem 8. An extended example in $\S 5$ and final comments conclude the paper. 


\section{Determinantal forms in $e_{\lambda}$ and $m_{\lambda}$ functions}

\section{Lemma 1}

Let

$$
J_{\rho}=\sum_{\kappa} j_{\rho \kappa}^{m} m_{\kappa}=\sum_{\kappa} j_{\rho \kappa}^{e} e_{\kappa}
$$

Then $j_{\rho \kappa}^{m}=0$ unless $\rho \geq \kappa ;$ and $j_{\rho \kappa}^{e}=0$ unless $\kappa \geq \rho^{\prime}$. Moreover $j_{\rho \rho}^{m} \neq 0$ and $j_{\rho \rho^{\prime}}^{e} \neq 0$.

\section{Proof 1}

The statements for the $m_{\lambda}$ functions are well-known (e.g. [4, pp. 326, 379], [6, p. 77]). The conclusions for the $e_{\lambda}$ functions then follow directly by noting (from [7, p. 43] or [4, p. 20], i.a.) that, for suitable constants $v_{\kappa \sigma}$ :

$$
m_{\kappa}=e_{\kappa}+\sum_{\sigma>\kappa^{\prime}} v_{\kappa \sigma} e_{\sigma}
$$

The proof of Lemma 2 is straightforward (e.g. [5, §4.4]).

\section{Lemma 2}

If $\kappa>\lambda$, then there is a chain $\kappa=\kappa_{0}>\kappa_{1}>\kappa_{2} \ldots>\kappa_{t}=\lambda$, such that adjacent partitions differ only in 2 elements, and these elements differ by unity. That is, for each $q$,

$$
\kappa_{q}=\left(k_{1}, \ldots, k_{i}, \ldots, k_{j}, \ldots\right) \quad \text { and } \quad \kappa_{q+1}=\left(k_{1}, \ldots, k_{i}-1, \ldots, k_{j}+1, \ldots\right)
$$

where the entries other than the ith and $j$ th are unaltered.

\section{Theorem 3}

Let $w(\kappa)=w(\lambda)$. Then

$$
\kappa>\lambda \Rightarrow c_{\kappa}>c_{\lambda} \Leftrightarrow d_{\kappa}>d_{\lambda}
$$

\section{Proof 3}

With notation as in Lemma 2 , it is easy to show from (8) that $c_{\kappa_{q}}>c_{\kappa_{q+1}}$, after which a glance at (9) completes the proof.

The following development uses the $\mathcal{L}$ operator and the eigenvalue $c_{\lambda}$. There would be no difference to Theorem 4 if one were instead to use $\mathcal{H}$ and $d_{\lambda}$ in this treatment. The diagonal elements in the resulting determinants are $c_{\rho}-c_{\lambda}$, which $\equiv d_{\rho}-d_{\lambda}$ from (9), while off diagonal elements would be unaltered. 
Only the expansion in $e_{\lambda}$ functions is derived. The analogous expansion in $m_{\lambda}$ functions duplicates the result derived in [3], and both expansions are listed in Theorem 4.

Let $J_{\rho}=\sum_{\kappa} j_{\rho \kappa}^{e} e_{\kappa}=j^{T} E$ in an obvious notation. Then

$$
\mathcal{L} J_{\rho}=\mathcal{L} j^{T} E=j^{T} \mathcal{L} E=j^{T} \Omega^{l e} E .
$$

But from (4) one has

$$
\mathcal{L} J_{\rho}=c_{\rho} J_{\rho}=c_{\rho} j^{T} E
$$

from which

$$
j^{T}\left(\Omega^{l e}-c_{\rho} I\right) E=0,
$$

where $I$ denotes the identity matrix. The $e_{\lambda}$ functions are functionally independent, so that

$$
j^{T}\left(\Omega^{l e}-c_{\rho} I\right)=0 .
$$

Now, from Lemma $1, j_{\rho \kappa}^{e}=0$ unless $\kappa \geq \rho^{\prime}$. In the matrix $\Omega^{l e}-c_{\rho} I$, we therefore omit all rows and columns indexed by partitions $\sigma$ such that

$$
\rho^{\prime}>^{R} \sigma ; \quad \text { or } \quad \sigma \stackrel{R}{>}^{\prime} \text { and } \sigma \ngtr \rho^{\prime} .
$$

The matrix resulting from these deletions is $\Omega_{\rho, 0}^{e}$. It is lower triangular, with zero in the last diagonal element, but no further zeroes along the diagonal, by virtue of Theorem 3. The vector of coefficients $j$ is likewise reduced, albeit without change of notation; consistent with this, the vector $E$ is truncated, also without changing notation.

In fact the last column of $\Omega_{\rho, 0}^{e}$ is zero. One can utilise the final diagonal element to normalise $J_{\lambda}$, or one can insert $E$ into that vacuous final column. Setting $z$ to be a column vector of zeroes save for unity in the final position, we have respectively:

$$
j^{T} \Omega_{\rho, 2}^{e}=j^{T}\left(\Omega_{\rho, 0}^{e}+(0 \mid z)\right)=(0,0,0, \ldots, 0, N)=N z^{T}
$$

where $j_{\rho \rho^{\prime}}^{e}=N$ and the 0 in $(0 \mid z)$ is a zero matrix of the appropriate order; and

$$
j^{T} \Omega_{\rho, 1}^{e}=j^{T}\left(\Omega_{\rho, 0}^{e}+(0 \mid E)\right)=J_{\rho} z^{T} .
$$

With a non-zero final diagonal element, the matrices $\Omega_{\rho, 1}^{e}$ and $\Omega_{\rho, 2}^{e}$ are non-singular from Theorem 3, and

$$
j^{T}=N z^{T}\left(\Omega_{\rho, 2}^{e}\right)^{-1}=J_{\rho} z^{T}\left(\Omega_{\rho, 1}^{e}\right)^{-1} .
$$

The two inverse matrices have proportional final rows, since the cofactors are identical. Therefore

$$
J_{\rho}=\frac{N}{\operatorname{det}\left(\Omega_{\rho, 2}^{e}\right)} \operatorname{det}\left(\Omega_{\rho, 1}^{e}\right) .
$$


A similar development in $m_{\lambda}$ functions serves to corroborate the results in [3]. Combining the two results yields the following theorem, and examples of the expansions are provided in $\S 5$.

\section{Theorem 4}

Using the above notation we have

$$
J_{\rho}=\frac{j_{\rho \rho^{\prime}}^{e}}{\operatorname{det}\left(\Omega_{\rho, 2}^{e}\right)} \operatorname{det}\left(\Omega_{\rho, 1}^{e}\right)=\frac{j_{\rho \rho}^{m}}{\operatorname{det}\left(\Omega_{\rho, 2}^{m}\right)} \operatorname{det}\left(\Omega_{\rho, 1}^{m}\right),
$$

in which the definitions of $\Omega_{\rho, 1}^{m}$ and $\Omega_{\rho, 2}^{m}$ are analogous to those of $\Omega_{\rho, 1}^{e}$ and $\Omega_{\rho, 2}^{e}$.

\section{Symmetry between the operator matrices for $m_{\lambda}$ and $e_{\lambda}$ functions}

A referee's suggestion to use Macdonald's $\omega_{\alpha}$ operator in the proof of Lemma 7 shortened the paper considerably.

\section{Theorem 5}

Define operator matrices $\Omega$ as follows:

$$
\mathcal{L}^{(\alpha)} M=\Omega^{l m}(\alpha) M \quad \mathcal{L}^{(\alpha)} E=\Omega^{l e}(\alpha) E
$$

and break them up into diagonal and off-diagonal portions

$$
\Omega^{l m}(\alpha)=D^{l m}(\alpha)+\Omega_{u}^{m}(\alpha) \quad \Omega^{l e}(\alpha)=D^{l e}(\alpha)+\Omega_{l}^{e}(\alpha),
$$

where $D$ matrices are diagonal; and $\Omega_{l}^{e}(\alpha)$ and $\Omega_{u}^{m}(\alpha)$ have zero diagonals.

Then $\Omega_{u}^{m}$ is upper triangular, and does not depend on $\alpha$. Moreover

$$
\Omega_{l}^{e}(\alpha)=-\alpha \Omega_{u}^{m} T .
$$

\section{Proof 5}

As already discussed just after equation (5), the matrix $\Omega^{h m}$ is known from [3]. Given that discussion, the proof is immediate from Lemma 7, noting that the terms involving the identity matrix in (16) and (17) naturally have no impact off the diagonal.

\section{Lemma 6}

$$
-\alpha \mathcal{L}^{(1 / \alpha)}=\omega_{\alpha} \mathcal{L}^{(\alpha)} \omega_{1 / \alpha}-(1+\alpha) k_{1}
$$


and

$$
-\alpha \mathcal{H}^{(1 / \alpha)}=\omega_{\alpha} \mathcal{H}^{(\alpha)} \omega_{1 / \alpha}-(1+\alpha)\left(k_{1}+k_{2}\right)
$$

where $k_{1}=w(n-1)$ and $k_{2}=w(\alpha-n+1) / 2$. The operator $\omega_{\alpha}$ is defined in (19).

\section{Proof 6}

In $\left[4\right.$, p. 320] Macdonald defines an operator $\square^{\alpha}$, which he calls the Laplace Beltrami operator, as

$$
\square^{\alpha}=\mathcal{L}^{(\alpha)}-w(n-1)=\mathcal{L}^{(\alpha)}-k_{1} .
$$

From [4, p 330, Ex. 3] one has

$$
\omega_{\alpha} \square^{\alpha}+\alpha \square^{1 / \alpha} \omega_{\alpha}=0,
$$

or equivalently

$$
-\alpha \square^{1 / \alpha}=\omega_{\alpha} \square^{\alpha} \omega_{1 / \alpha} .
$$

Now the left side of (14) becomes

$$
-\alpha \mathcal{L}^{(1 / \alpha)}=-\alpha\left(\square^{1 / \alpha}+k_{1}\right)=\omega_{\alpha} \square^{\alpha} \omega_{1 / \alpha}-k_{1} \alpha=\omega_{\alpha} \mathcal{L}^{(\alpha)} \omega_{1 / \alpha}-k_{1}(1+\alpha),
$$

agreeing with the right side.

From (3) we have that $\mathcal{H}-\mathcal{L}=k_{2}$, so that $\square^{\alpha}-\mathcal{H}=-k_{1}-k_{2}$, and the second result (15) is proved similarly.

\section{Lemma 7}

$$
\Omega^{l e}(\alpha)=-\alpha \Omega^{l m}(1 / \alpha)^{T}-k_{1}(1+\alpha) I
$$

and

$$
\Omega^{h e}(\alpha)=-\alpha \Omega^{h m}(1 / \alpha)^{T}-\left(k_{1}+k_{2}\right)(1+\alpha) I .
$$

\section{Proof 7}

We take as given the inner product $\langle., .\rangle_{\alpha}$ used to define the Jack polynomials, with respect to which they are mutually orthogonal (see [6, p. 77], [4, Ch. VI, §§1, 10]). The treatment here and notation follow that in [4, p. 378].

With respect to $\langle., .\rangle_{\alpha}$, the set of functions $\left\{g_{\mu}^{(\alpha)}\right\}$ are defined as those which are dual to the $m_{\lambda}$ functions. That is,

$$
\left\langle g_{\mu}^{(\alpha)}, m_{\lambda}\right\rangle_{\alpha}=\delta_{\mu \lambda} .
$$

The operator $\omega_{\alpha}$ is defined as

$$
\omega_{\alpha} g_{\mu}^{(\alpha)}=e_{\mu}
$$


and satisfies the following identity:

$$
\omega_{\alpha} \omega_{1 / \alpha}=1
$$

Having listed the properties of the operator $\omega_{\alpha}$ we proceed to the proof itself. From (12) we may write

$$
\mathcal{L}^{(\alpha)} m_{\kappa}=\sum_{\rho}\left(\Omega^{l m}(\alpha)\right)_{\kappa \rho} m_{\rho}
$$

The fact that the $\mathcal{L}^{(\alpha)}$ operator is self adjoint with respect to the inner product $\langle., .\rangle_{\alpha}$ (see e.g. [8, p. 112]) allows us to write

$$
\left\langle\mathcal{L}^{(\alpha)} g_{\lambda}^{(\alpha)}, m_{\kappa}\right\rangle_{\alpha}=\left\langle g_{\lambda}^{(\alpha)}, \mathcal{L}^{(\alpha)} m_{\kappa}\right\rangle_{\alpha}=\left(\Omega^{l m}(\alpha)\right)_{\kappa \lambda},
$$

from which the following is immediate:

$$
\mathcal{L}^{(\alpha)} g_{\lambda}^{(\alpha)}=\sum_{\kappa}\left(\Omega^{l m}(\alpha)\right)_{\kappa \lambda} g_{\kappa}^{(\alpha)}
$$

The relation (19) and Lemma 6 imply

$$
-\alpha \mathcal{L}^{(1 / \alpha)} e_{\lambda}=\omega_{\alpha} \mathcal{L}^{(\alpha)} \omega_{1 / \alpha} e_{\lambda}-k_{1}(1+\alpha) e_{\lambda}=\omega_{\alpha} \mathcal{L}^{(\alpha)} g_{\lambda}^{(\alpha)}-k_{1}(1+\alpha) e_{\lambda} .
$$

From (23) and (19), we have

$$
\begin{gathered}
-\alpha \mathcal{L}^{(1 / \alpha)} e_{\lambda}=\omega_{\alpha} \sum_{\kappa}\left(\Omega^{l m}(\alpha)\right)_{\kappa \lambda} g_{\kappa}^{(\alpha)}-k_{1}(1+\alpha) e_{\lambda} \\
=\sum_{\kappa}\left(\Omega^{l m}(\alpha)\right)_{\kappa \lambda} e_{\kappa}-k_{1}(1+\alpha) e_{\lambda} .
\end{gathered}
$$

Interchanging $\alpha$ and $1 / \alpha$, this expression is equivalent to

$$
\mathcal{L}^{(\alpha)} e_{\lambda}=-\alpha \sum_{\kappa}\left(\Omega^{l m}(1 / \alpha)\right)_{\kappa \lambda} e_{\kappa}-k_{1}(1+\alpha) e_{\lambda} \equiv \sum_{\kappa}\left(\Omega^{l e}(\alpha)\right)_{\lambda \kappa} e_{\kappa},
$$

whence (16) follows. The proof of (17) is similar.

\section{The action of $\mathcal{L}$ on the $e_{\lambda}$ functions}

The final technical point, as noted in $\S 1.2$, is to show that the coefficient of $e_{\lambda}$ in (6) is indeed $c_{\lambda^{\prime}}$. 


\section{Theorem 8}

$$
\mathcal{L} e_{\lambda}=c_{\lambda^{\prime}} e_{\lambda}+\sum_{\kappa>\lambda} b_{\lambda \kappa} e_{\kappa}
$$

in which the coefficients $b_{\lambda \kappa}$ are given in Theorem 5 .

\section{Proof 8}

Let

$$
\mathcal{L} e_{\lambda}=f_{\lambda} e_{\lambda}+\sum_{\kappa>\lambda} b_{\lambda \kappa} e_{\kappa}
$$

From Lemma 1 one has

$$
J_{\lambda}=j_{\lambda \lambda^{\prime}}^{e} e_{\lambda^{\prime}}+\sum_{\rho>\lambda^{\prime}} j_{\lambda \rho}^{e} e_{\rho}
$$

Then

$$
\begin{gathered}
\mathcal{L} J_{\lambda}=j_{\lambda \lambda^{\prime}}^{e} \mathcal{L} e_{\lambda^{\prime}}+\sum_{\rho>\lambda^{\prime}} j_{\lambda \rho}^{e} \mathcal{L} e_{\rho} \\
=j_{\lambda \lambda^{\prime}}^{e}\left(f_{\lambda^{\prime}} e_{\lambda^{\prime}}+\sum_{\rho>\lambda^{\prime}} b_{\lambda^{\prime} \rho} e_{\rho}\right)+\sum_{\rho>\lambda^{\prime}} j_{\lambda \rho}^{e}\left(f_{\rho} e_{\rho}+\sum_{\sigma>\rho} b_{\rho \sigma} e_{\sigma}\right) \\
=j_{\lambda \lambda^{\prime}}^{e} f_{\lambda^{\prime}} e_{\lambda^{\prime}}+\sum_{\kappa>\lambda^{\prime}} q_{\lambda \kappa} e_{\kappa} .
\end{gathered}
$$

But from (4) and Lemma 1 we have

$$
\mathcal{L} J_{\lambda}=c_{\lambda} J_{\lambda}=c_{\lambda}\left(j_{\lambda \lambda^{\prime}}^{e} e_{\lambda^{\prime}}+\sum_{\rho>\lambda^{\prime}} j_{\lambda \rho}^{e} e_{\rho}\right)
$$

Recalling that $j_{\lambda \lambda^{\prime}}^{e} \neq 0$, one sees that $c_{\lambda^{\prime}}=f_{\lambda}$.

\section{Extended example}

For partitions of weight 4:

$$
\Omega^{l m}=\left(\begin{array}{ccccc}
c_{(4)} & 4 & 4 & & \\
& c_{(3,1)} & 2 & 6 & \\
& & c_{(2,2)} & 2 & \\
& & & c_{(2,1,1)} & 12 \\
& & & & c_{(1,1,1,1)}
\end{array}\right)
$$




$$
\begin{aligned}
& \Omega_{(4), 0}^{m}=\left(\begin{array}{ccccc}
0 & 4 & 4 & & \\
& c_{(3,1)}-c_{(4)} & 2 & 6 & \\
& & c_{(2,2)}-c_{(4)} & 2 & \\
& & & c_{(2,1,1)}-c_{(4)} & 12 \\
& & & & c_{(1,1,1,1)}-c_{(4)}
\end{array}\right) \\
& J_{(4)}=\left|\begin{array}{ccccc}
m_{(4)} & 4 & 4 & & \\
m_{(3,1)} & -1-3 \alpha & 2 & 6 & \\
m_{(2,2)} & & -2-4 \alpha & 2 & \\
m_{(2,1,1)} & & & -3-5 \alpha & 12 \\
m_{(1,1,1,1)} & & & & -6-6 \alpha
\end{array}\right|
\end{aligned}
$$

The above example conforms with the example given in [3].

$$
\begin{gathered}
\Omega_{(2,2), 0}^{m}=\left(\begin{array}{ccc}
0 & 2 & \\
& c_{(2,1,1)}-c_{(2.2)} & 12 \\
& & c_{(1,1,1,1)}-c_{(2,2)}
\end{array}\right) \\
J_{(2,2)}=\left|\begin{array}{ccc}
m_{(2,2)} & 2 & 12 \\
m_{(2,1,1)} & -1-\alpha & -4-2 \alpha \\
m_{(1,1,1,1)} & -4
\end{array}\right|
\end{gathered}
$$

Turning to the $e_{\lambda}$ basis functions,

$$
\begin{aligned}
& \Omega^{l e}=\left(\begin{array}{ccccc}
c_{(1,1,1,1)} & & & & \\
-4 \alpha & c_{(2,1,1)} & & & \\
-4 \alpha & -2 \alpha & c_{(2,2)} & & \\
& -6 \alpha & -2 \alpha & c_{(3,1)} & \\
& & & -12 \alpha & c_{(4)}
\end{array}\right) \\
& J_{(4)}=\left|\begin{array}{ccccc}
-6-6 \alpha & & & & e_{(4)} \\
-4 \alpha & -3-5 \alpha & & & e_{(3,1)} \\
-4 \alpha & -2 \alpha & -2-4 \alpha & & e_{(2,2)} \\
& -6 \alpha & -2 \alpha & -1-3 \alpha & e_{(2,1,1)} \\
& & & -12 \alpha & e_{(1,1,1,1)}
\end{array}\right| \\
& \Omega_{(3,1), 0}^{e}=\left(\begin{array}{cccc}
c_{(1,1,1,1)}-c_{(3,1)} & & & \\
-4 \alpha & c_{(2,1,1)}-c_{(3,1)} & & \\
-4 \alpha & -2 \alpha & c_{(2,2)}-c_{(3,1)} & \\
& -6 \alpha & -2 \alpha & 0
\end{array}\right) \\
& J_{(3,1)}=\left|\begin{array}{cccc}
-5-3 \alpha & & & e_{(4)} \\
-4 \alpha & -2-2 \alpha & & e_{(3,1)} \\
-4 \alpha & -2 \alpha & -1-\alpha & e_{(2,2)} \\
& -6 \alpha & -2 \alpha & e_{(2,1,1)}
\end{array}\right|
\end{aligned}
$$




\section{Concluding comment}

Roberts [5, p. 41] has shown that the proportion $p$ of non-zero elements in $\Omega^{l m}$ behaves as

$O\left(w^{3} \exp (-\pi \sqrt{2 w / 3})\right)$ as $w \rightarrow \infty$. The abbreviated matrices $\Omega_{\lambda, 0}^{m}$ and $\Omega_{\lambda, 0}^{e}$ will have a higher proportion $p$, simply because omitted rows and columns contain only zeroes with the general exception of the diagonal element.

For given $\lambda$, let $q$ denote the proportion of partitions $\kappa \stackrel{R}{<} \lambda$ such that $w(\kappa)=w(\lambda)$ and $\kappa \nless \lambda$. From casual observation, for small $w$ one would say that $q$ is usually small, even for $\lambda$ high in the RLO; but for large $w$ the value of $q$ is likely to be much higher, especially when $\lambda$ is high in the RLO. Nevertheless one expects $\Omega_{\lambda, 0}^{m}$ and $\Omega_{\lambda, 0}^{e}$ to be sparse in practice.

\section{References}

[1] S Helgason. Differential Geometry and Symmetric Spaces. Academic Press, 1962.

[2] A T James. Calculation of zonal polynomial coefficients by use of the Laplace Beltrami operator. Annals of Mathematical Statistics, 39:1711-1718, 1968.

[3] L Lapointe, A Lascoux, and J Morse. Determinantal expression and recursion for jack polynomials. Electronic Journal of Combinatorics, 7(1), 2000.

[4] I G Macdonald. Symmetric Functions and Hall Polynomials. Clarendon Press, 2nd edition, 1995.

[5] L A Roberts. On the expansion of zonal polynomials in monomial symmetric functions. Technical Report 98-31, School of Mathematical and Computing Sciences, Victoria University, Wellington, New Zealand, December 1998.

[6] R P Stanley. Some combinatorial properties of Jack symmetric functions. Advances in Mathematics, 77:76-115, 1989.

[7] A Takemura. Zonal Polynomials, volume 4 of Lecture Notes - Monograph Series. Institute of Mathematical Statistics, 1984.

[8] N Ja Vilenkin and A U Klimyk. Representation of Lie Groups and Special Functions: Recent Advances, volume 316 of Mathematics and its Applications. Kluwer Academic Publishers, 1995. 